BULL. AUSTRAL. MATH. SOC.

VOL. 7 (1972), 163-168.

\title{
Pseudo-fields and doubly transitive groups
}

\author{
F.W. Wilke
}

A sharply doubly transitive group which acts on a set of at least two elements is isomorphic to the group of affine transformations on a system $S$. This statement is true if $S$ is replaced by either strong pseudo-field or pseudo-field. The additive system of a strong pseudo-field is a loop while the additive system of a pseudo-field need not be a loop. We show that any pseudo-field is either a strong pseudo-field or can be obtained from a strong pseudo-field in a nice way. Every near-field is a strong pseudo-field. The converse is an open question.

DEFINITION 1 (Tits, [2]). A (left) pseudo-field $(F,+, \cdot)$ is a set $F$ of cardinality at least two with binary operations + and $\cdot$ such that

(1) there exists $0 \in F$ such that $x+0=0+x=x$ and $x \cdot 0=0 \cdot x=0$ for all $x \in F$;

(2) for each $a \in F$ there exists $-a \in F$ such that $a+(-a)=(-a)+a=0$;

(3) $F-\{0\}$ is a group under - with identity 1 ;

(4) $x \cdot(y+z)=x \cdot y+x \cdot z$ for all $x, y, z \in F$;

(5) for $a, b \in F$ there exists an element $\rho(a, b) \in F$ such that $(x+a)+b=\rho(a, b) \cdot x+(a+b)$ for all $x \in F$. 
THEOREM 1 (Tits, [2]). A shorply doubly transitive group of permutations acting on a set of cardinality at least two is isomorphic to the group of affine transformations $x \rightarrow a \cdot x+b$ on a pseudo-field.

DEFINITION 2. A (left) near-field $(N,+, \cdot)$ is a system of cardinality at least two such that $(N,+)$ is an abelian group with identity $0, N-\{0\}$ is a group under - with identity $1,0 \cdot x=0$ for all $x \in N$ and $x \cdot(y+z)=x \cdot y+x \cdot z$ for all $x, y, z \in N$. If, in addition, $r, s, t \in N$ with $r \neq s$ implies there exists $x \in N$ such that $r \cdot x=s \cdot x+t$, then $(N,+, \cdot)$ is called a planar near-field.

In [1, Theorem 20.7.1] Hall proves

THEOREM 2. If $G$ is a shorply doubly transitive group of permutations acting on a set $E$ of cardinality at least two such that either

(1) $E$ is finite or

(2) $i$ and $j$ distinct elements of $E$ implies there exists at most one $g \in G$ such that $g(i)=j$ and $g$ has no fixed point

then $G$ is isomorphic to the group of affine transformations $x \rightarrow a \cdot x+b$ on a planar near-fieza.

Zemmer [3] has constructed a class of non-planar near-fields. The group of affine transformations on such a near-field is sharply doubly transitive but does not satisfy either (1) or (2) of Theorem 2.

Suppose $G$ is a permutation group acting on a set $E$ which satisfies the conditions of Theorem 2. Let 0,1 be distinct elements of $E$ and let $G_{0}$ be the stabilizer of 0 . For each $i \in E, i \neq 0$, let $g_{i}$ be the unique element of $G$ such that $g_{i}(0)=i$ and $g_{i}(k) \neq k$ for all $k \in E$ and let $m_{i}$ be the unique element of $G_{0}$ such that $m_{i}(1)=i$. Finally, let $g_{0}$ be the identity. Then Hall shows that $(E,+, \cdot)$ is a planar near-field where + and - are defined by

$$
x+y=g_{y}(x)
$$

end 


$$
x \cdot y= \begin{cases}0 & \text { if } x=0, \\ m_{x}(y) & \text { if } x \neq 0 .\end{cases}
$$

The group of affine transformations on this near-field is isomorphic to the group $G$.

Even without assuming (1) and (2) of Theorem 2 an element of $G$ may be chosen to play the role of $g_{y}$ as follows. For each $x \in E, x \neq 0$, let $t_{x}$ be the unique involution which maps 0 onto $x$. In any doubly transitive group the involutions occur in a single conjugate class. Thus, ei ther

(a) each involution has a unique fixed point and $t_{0}$ is defined to be the unique involution fixing 0 or

(b) no involution has a fixed point and $t_{0}$ is defined to be the identity.

Then, if + is defined by

$$
x+y=t_{y} t_{0}(x)
$$

and $\cdot$ is defined as above then $(E,+, \cdot)$ is a system called a strong pseudo-field, and $G$ is isomorphic to the group of affine transformations $x \rightarrow a \cdot x+b$ on $(E,+, \cdot)$.

DEFINITION 3. A strong pseudo-field $(F,+, \cdot)$ is a set $F$ of cardinality at least two such that

(1) $(F,+)$ is a loop with identity 0 ;

(2) $E-\{0\}$ is a group under - with identity 1 ;

(3) $x \cdot(y+z)=x \cdot y+x \cdot z$ for all $x, y, z \in F$;

(4) $0 \cdot x=0$ for all $x \in F$;

(5) for $a, b \in F$ there exists an element $\rho(a, b) \in F$ such that $(x+a)+b=\rho(a, b) \cdot x+(a+b)$ for all $x \in F$.

It is imnediate that a near-field is a strong pseudo-field which, in turn, is a pseudo-field. Example 1 (see $[2,5.6]$ ) below, shows that there 
are pseudo-fields which are not strong pseudo-fields. It is not known whether or not there exist strong pseudo-fields which are not near-fields.

EXAMPLE 1. Let $(F,+, \cdot)$ be a strong pseudo-field in which $1+1 \neq 0$. Define

$$
x \oplus y=\left\{\begin{array}{l}
-x+y \text { if } y \neq 0, \\
x+y \text { if } y=0 .
\end{array}\right.
$$

Then $(F, \Theta, \cdot)$ is a pseudo-field but is not a strong pseudo-field since $(F, \Theta)$ is not a loop. In particular, for any $a \neq 0$ the equation $a \oplus x=-a$ has no solution. On the other hand, in any pseudo-field,

$$
-a=((-a)+a)+(-a)=p(a,-a) \cdot(-a)+(a+(-a))=p(a,-a) \cdot(-a),
$$

so that $\rho(\alpha,-a)=1$. Thus $(x+a)+(-a)=x$ for all $x, a \in F$ and the equation $x+a=b$ has the solution $x=b+(-a)$. Also, since $0=(-1) \cdot[1+(-1)]=(-1)+(-1) \cdot(-1)$ we have $(-1) \cdot(-1)=1$ in any pseudo-field.

THEOREM 3. If $(F,+, \cdot)$ is a pseudo-field then $(F,+, \cdot)$ is a strong pseudo-field or may be obtained from a strong pseudo-field by the procedure in Example 1 .

Proof. Let $(F,+, \cdot)$ be a pseudo-field. For $a, b \in F$ with $a \neq 0$ let $T_{a, b}$ be the permutation of $F$ given by $T_{a, b}(x)=a \cdot x+b$. If $G=\left\{T_{a, b} \mid a, b \in F, a \neq 0\right\}$ then $G$ is a sharply doubly transitive group acting on $F$; (see $[2,5.3]$ ). Let $G_{0}$ be the stabilizer of 0 . Each element of $G_{0}$ is of the form $T_{a, 0}$ for $a \in F, a \neq 0$. For each $x \in F, x \neq 0$, let $t_{x}$ be the unique involution in $G$ which interchanges 0 and $x$. If the involutions of $G$ have fixed points then $t_{0}$ is the unique involution fixing 0 . Otherwise $t_{0}$ is the identity mapping. Define $x \oplus y=t_{y} t_{0}(x)$. As noted above, $(F, \oplus, \cdot)$ is a strong pseudo-field.

Suppose the involutions of $G$ have no fixed points. If $a \cdot a=1$ then $T_{a, 0} T_{a, 0}=T_{I, 0}$. Thus $a=1$, since otherwise $T_{a, 0}$ is an involution fixing 0 . Since $(-1) \cdot(-1)=1$ we see that $-x=x$ and 
$x+x=0$ for all $x \in F$. Therefore, $T_{1, x_{1, x}}(z)=(z+x)+x=z$ since $\rho(x,-x)=\rho(x, x)=1$. Thus, $t_{x}=T_{1, x}$ and

$$
z+x=T_{1, x}(z)=t_{x} t_{0}(z)=z \oplus x
$$

for all $z, x \in F$. Therefore, if the involutions of $G$ have no fixed points then $(F,+, \cdot)$ is a strong pseudo-field.

Suppose the involutions of $G$ have fixed points. Then $t_{0}=T_{a, 0}$ for some $a \in F$ such that $a \cdot a=1$. Since $t_{0}$ is not the identity mapping, $a \neq 1$. In. fact, $a$ is the unique element of $F$ of multiplicative order two. Since $(-1) \cdot(-1)=1$ we must have $-1=a$ or $-1=1$.

$$
\text { If } \begin{aligned}
-1 & =a \text { then } T_{-1, y}=t_{y} \text { and } \\
& x+y=T_{1, y}(x)=T_{-1, y^{T}-1,0}(x)=t_{y} t_{0}(x)=x \oplus y,
\end{aligned}
$$

and $(F,+, \cdot)$ is a strong pseudo-field.

$$
\text { If }-1=1 \text { then } x+x=0 \text { for all } x \in F \text { and } T_{1, x}=t_{x} \text { if }
$$

$x \neq 0$. Thus

$$
\begin{aligned}
x+y & =T_{1, y}(x)=T_{1, y^{T} a, 0} T_{a, 0}(x) \\
& = \begin{cases}t_{y} t_{0}(a \cdot x) & \text { if } y \neq 0, \\
x & \text { if } y=0,\end{cases} \\
& = \begin{cases}(a \cdot x) \oplus y & \text { if } y \neq 0, \\
x & \text { if } y=0 .\end{cases}
\end{aligned}
$$

Since for $x \neq 0,0=x+x=a \cdot x \oplus x$ we have $a \cdot x=$ Ox where $\theta$ denotes the negative with respect to $\oplus$. Therefore,

$$
x+y= \begin{cases}\Theta x \oplus y & \text { if } y \neq 0, \\ x & \text { if } y=0,\end{cases}
$$

so that $(F,+, \cdot)$ can be obtained from the strong pseudo-field $(F, \oplus, \cdot)$ by the procedure in Example 1 . 
To give the reader some idea of how "close" a strong pseudo-field is to being a near-field we list, without proof, some properties of the additive loop $(F,+)$ of a strong pseudo-field.

1. $(F,+)$ is a right Bol loop.

2. $(F,+)$ has the automorphic inverse property.

3. (F, +) has a sharply simply transitive group of automorphisms.

4. If $(E,+)$ satisfies any one of the properties - left inverse, weak inverse, crossed inverse - then $(F,+)$ is an abelian group and hence $(F,+, \cdot)$ is a near-field.

\section{References}

[1] Marshall Hall, Jr, The theory of groups (The Macmilian Company, New York, 1959 ).

[2] J. Tits, "Sur les groupes doublement transitifs continus", Comment. Math. Helv. 26 (1952), 203-224.

[3] J.T. Zemmer, "Near-fields, planar and non-planar", Math. Student 32 (1964), 145-150.

Department of Mathematics,

University of Missouri - St Louis,

St Louis,

Missouri,

USA. 